\title{
Blind Sac Approach Using Silastic Block for Cochlear Implantation in Patients with Cholesteatoma
}

\author{
Ah-Ra Lyu ${ }^{1}$ and Yong-Ho Park ${ }^{2,3}$ \\ ${ }^{1}$ Department of Medical Science, College of Medicine, Chungnam National University, Daejeon, \\ ${ }^{2}$ Department of Otolaryngology-Head and Neck Surgery, College of Medicine, Chungnam National University, Daejeon, \\ ${ }^{3}$ Brain Research Institute, College of Medicine, Chungnam National University, Daejeon, Korea
}

Received August 1, 2017

Revised August 22, 2017

Accepted August 22, 2017
Cochlear implant $(\mathrm{Cl})$ surgery in cholesteatoma is challenging because of the risk of residual or recurrent infection. Although $\mathrm{Cl}$ could be done with subtotal petrosectomy in single or staged surgery, this surgery needed additional surgical procedures to obliterate the mastoid cavity. This paper describes a new surgical technique for $\mathrm{Cl}$ surgery in cholesteatoma without external auditory canal closure.

J Audiol Otol 2017;21(3):152-155
Department of Otolaryngology-Head and Neck Surgery,

College of Medicine,

Chungnam National University,

282 Munwha-ro, Jung-gu,

Daejeon 35015, Korea

Tel +82-42-280-7697

Fax +82-42-253-4059

E-mail parkyh@cnu.ac.kr
KEY WORDS: Cochlear implant · Cholesteatoma $\cdot$ Infection

\section{Introduction}

Cochlear implantation (CI) in patients with chronic ear infection such as chronic suppurative otitis media or cholesteatoma can be done either through a single stage or multi-staged operation [1-3]. The foremost issue for these patients is hearing rehabilitation via successful implantation of a device with no risk of recurrent infection that may lead to explantation of the device. A non-infective or infection free ear is mandatory before CI and to achieve this, many surgeons prefer subtotal petrosectomy with mastoid obliteration and closure of the external auditory canal (EAC) [4,5]. However, some patients who underwent this surgery needed additional surgical procedures to retrieve the mastoid cavity obliteration materials such as abdominal fat or large temporalis muscle flap. Some were also dissatisfied with the cosmetic outcome after EAC closure.

We herewith report several cases of patients with cholesteatoma who received CI via a blind sac approach without EAC

This is an Open Access article distributed under the terms of the Creative Commons Attribution Non-Commercial License (http://creativecommons.org/licenses/by-nc/4.0/) which permits unrestricted non-commercial use, distribution, and reproduction in any medium, provided the original work is properly cited. closure and discuss the advantages of our method.

\section{Case Report}

A 51-year-man presented with bilateral hearing loss and repeated otorrhea on the left ear. He lost his hearing from the right ear since childhood. He has been using a hearing aid on the left ear for over 20 years. He started to notice decreasing benefits from the hearing aid on the left ear since 2 years ago. Hearing levels of both ears were almost out of scale. Otoscopic examination revealed that the state of the canal wall down mastoidectomy in both ears showed total adhesion with cholesteatoma. High resolution CT (HRCT) scans revealed recurred cholesteatoma in the left ear (Fig. 1). A staged CI surgery of the left ear was planned.

After the canal wall down mastoidectomy, all of the middle ear mucosa was removed and the Eustachian tube was blocked with bone dust and bone wax. In order to create enough neotympanum for the staged CI, a tympanomeatal flap obtained from the anterior wall of the EAC was elevated as far laterally as possible and about a $1.5 \times 1.0 \mathrm{~cm}$ round shaped silastic block was positioned in the middle ear (Fig. 2). The mastoid 
cavity was obliterated with retroauricular musculoperiosteal flap. Additional cartilage slices which were harvested from cavum conchae and temporalis muscle fascia were then positioned layer by layer with repositioning of the EAC skin (Fig. 3A).

Upon confirmation that wound healing has completed after the primary surgery (about 2 months after the primary surgery), a HRCT scan was done and revealed thick soft tissue density containing the musculocutaneous flap and cartilage slices on the mastoid and the lateralized tympanomeatal flap in the left ear (Fig. 4). The staged CI surgery was then done via retroauricular approach with elevation of the musculocutaneous flap over the mastoid. After silastic block removal, the CI was done through a round window approach and the middle ear cavity was then obliterated using the surrounding soft tissues and cartilage slices that were created during the

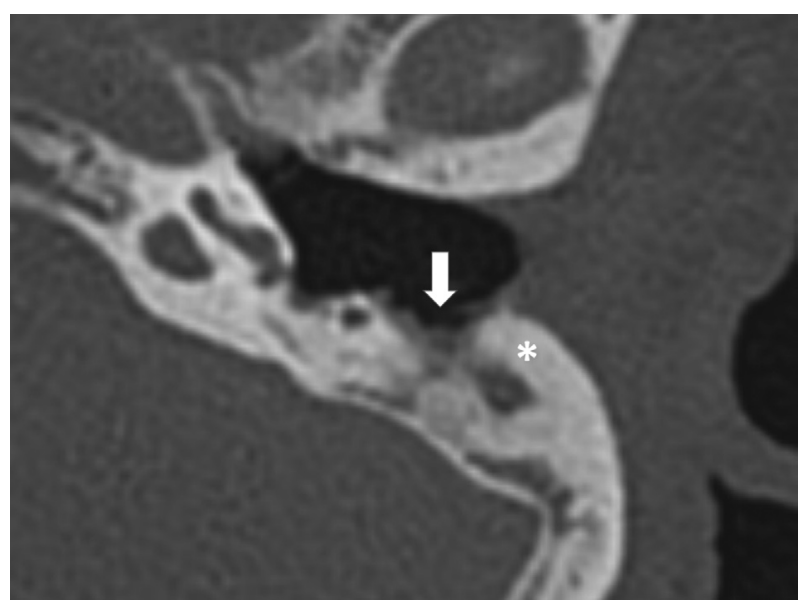

Fig. 1. High resolution computed tomography showing the state of the canal wall down mastoidectomy (white arrow) with new bone formation (asterisk) and severely atelectatic and adhesive ear drum in the left ear.

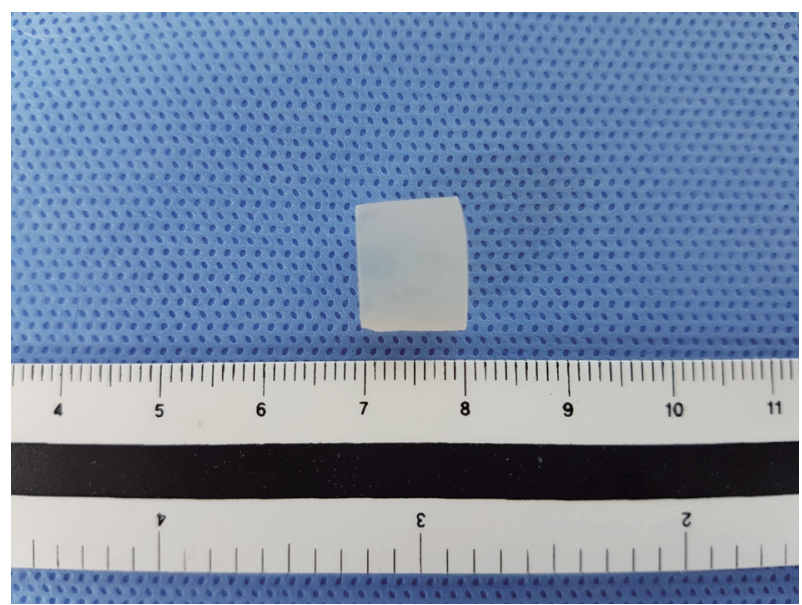

Fig. 2. Design of the silastic block (about $1.5 \mathrm{~cm}$ in width and $1 \mathrm{~cm}$ in height) that was used to create a neo-tympanum for the staged cochlear implantation. primary surgery. The electrode was also covered with the same cartilage slices followed by repositioning of the thick mastoid cavity flap (Fig. 3B).

Three patients with cholesteatoma underwent blind sac surgery with silastic block without EAC closure. There was no evidence of infection and electrode exposure after more than 2 years of follow up since the staged CI surgery (Fig. 5).

\section{Discussion}

When a CI surgery in chronic ear disease is needed, surgeons should be seriously aware of infection because uncontrolled or recurred infection can lead to wound infection which can result in the need for device explantation [3,6,7]. Regardless of the presence or absence of cholesteatoma, a clean and tolerable tympanum and mastoid cavity is mandatory before cochlear implantation.

A single stage CI surgery may be done successfully in ears without active infection and without cholesteatoma [1-3]. The primary goal of surgery in ears with active infection is the eradication of infectious foci and making the ear condition
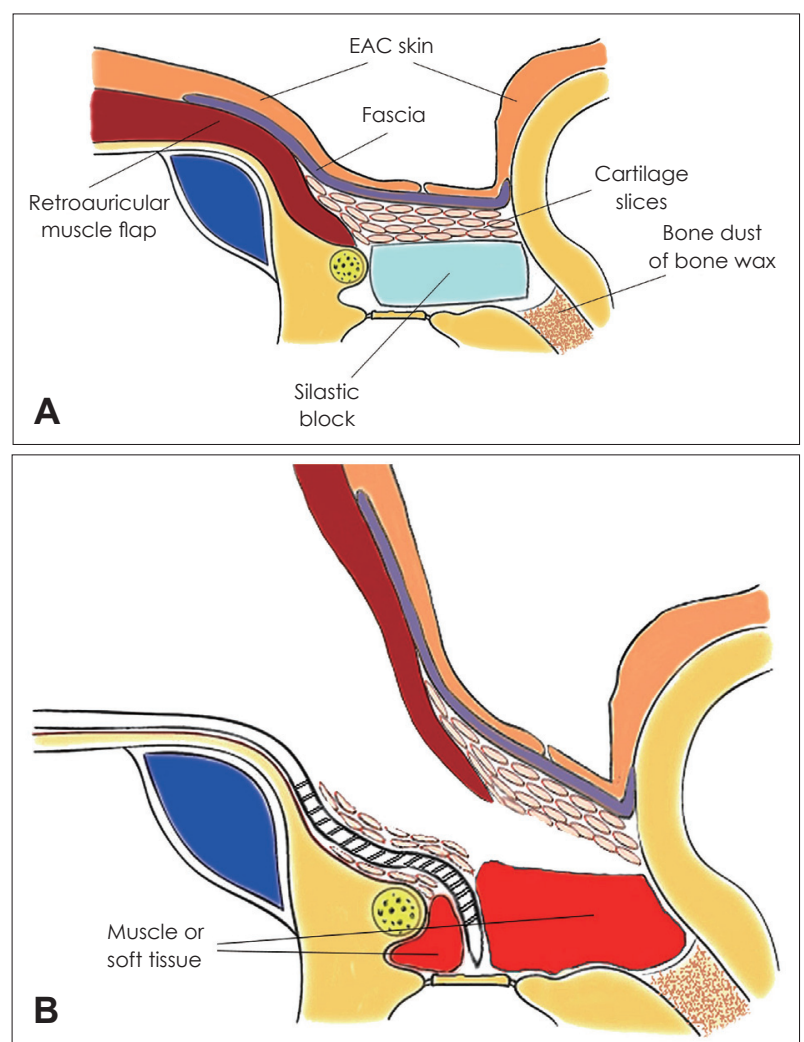

Fig. 3. Schematic illustration of surgery. Upon positioning of the silastic block, the mastoid was obliterated with retroauricular musculoperiosteal flap, cartilage slices, temporalis muscle fascia and lateralized external auditory canal skin flap (A). Staged cochlear implantation procedure after removal of the silastic block (B). EAC: external auditory canal. 


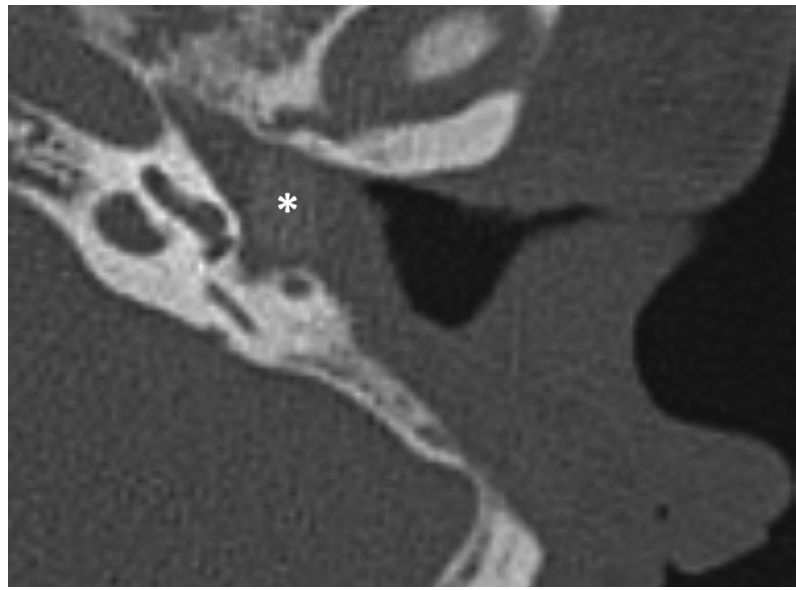

Fig. 4. High resolution computed tomography showing thick soft tissue with silastic block in the left mastoid and tympanum at 2 months after the primary surgery (asterisk).

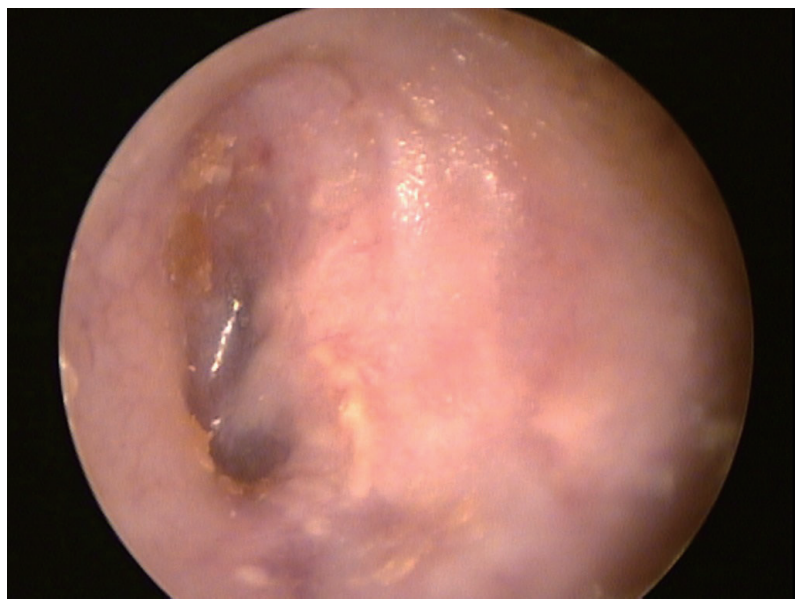

Fig. 5. Otoscopic finding of patient at 26 months after the staged cochlear implantation showing clear cavity without infection and no electrode exposure.

conducive (tympanum and mastoid) for implantation. These would be better and safely achieved through staged CI surgery than a single stage surgery $[1,3,8]$.

$\mathrm{CI}$ in cases with cholesteatoma should be managed very carefully because a number of patients with cholesteatoma have accompanying infection. Moreover, the potential of an existing biofilm to induce repeated infection even in cases of cholesteatoma without active infection is very real $[9,10]$. Many patients with cholesteatoma have Eustachian tube dysfunction which resulted in post-operative reperforation or severe retraction and adhesion of the eardrum. Although there were reports of single stage CI surgery bypassing the mastoid cavity $[11,12]$, a subtotal petrosectomy with EAC closure would be a good option for CI surgery in cholesteatoma [3-5,13-15]. Even with this surgery, there were several reports of cholesteatoma recurrence or break down of the EAC closure [1]. Fur- thermore, when the surgery is done with EAC closure, the detection of cholesteatoma recurrence can be difficult and additional surgery will be needed to retrieve the materials used for mastoid obliteration. Some patients were also dissatisfied with the cosmetic result because of the EAC closure.

We designed a surgical technique without EAC closure that required the creation of a suitable neo-tympanum and of an adequately sized thick mastoid skin flap to avoid electrode exposure. A silastic block was used to prevent retraction or adhesion of the ear drum and to create a neo-tympanum that is needed for the staged CI surgery. The silastic block was left in situ and occupied the tympanum after the primary surgery without developing any complication. It greatly aided in achieving a clean and adequate neo-tympanum. The fear of electrode exposure when EAC closure was not done was resolved via thick musculocutaneous flap and cartilage slices covering.

In conclusion, staged blind sac surgery with silastic block without EAC closure can be another option for CI surgery in patients with cholesteatoma.

\section{Acknowledgments}

This work was supported by the Basic Science Research Program through the National Research Foundation of Korea (NRF), funded by the Ministry of Education (NRF-2015R1D1A3A01018881 for Yong-Ho Park).

\section{Conflicts of interest}

The authors have no financial conflicts of interest.

\section{REFERENCES}

1) Basavaraj S, Shanks M, Sivaji N, Allen AA. Cochlear implantation and management of chronic suppurative otitis media: single stage procedure? Eur Arch Otorhinolaryngol 2005;262:852-5.

2) Jang JH, Park MH, Song JJ, Lee JH, Oh SH, Kim CS, et al. Longterm outcome of cochlear implant in patients with chronic otitis media: one-stage surgery is equivalent to two-stage surgery. J Korean Med Sci 2015;30:82-7.

3) Vincenti V, Pasanisi E, Bacciu A, Bacciu S, Zini C. Cochlear implantation in chronic otitis media and previous middle ear surgery: 20 years of experience. Acta Otorhinolaryngol Ital 2014;34:272-7.

4) Casserly P, Friedland PL, Atlas MD. The role of subtotal petrosectomy in cochlear implantation. J Laryngol Otol 2016;130 Suppl 4:S3540.

5) Free RH, Falcioni M, Di Trapani G, Giannuzzi AL, Russo A, Sanna M. The role of subtotal petrosectomy in cochlear implant surgery--a report of 32 cases and review on indications. Otol Neurotol 2013;34: 1033-40.

6) Farinetti A, Ben Gharbia D, Mancini J, Roman S, Nicollas R, Triglia JM. Cochlear implant complications in 403 patients: comparative study of adults and children and review of the literature. Eur Ann Otorhinolaryngol Head Neck Dis 2014;131:177-82.

7) Yoo MH, Park HJ, Yoon TH. Management options for cochlear implantation in patients with chronic otitis media. Am J Otolaryngol 2014;35:703-7.

8) Wong MC, Shipp DB, Nedzelski JM, Chen JM, Lin VY. Cochlear implantation in patients with chronic suppurative otitis media. Otol Neurotol 2014;35:810-4. 
9) Chole RA, Faddis BT. Evidence for microbial biofilms in cholesteatomas. Arch Otolaryngol Head Neck Surg 2002;128:1129-33.

10) Galli J, Calò L, Giuliani M, Sergi B, Lucidi D, Meucci D, et al. Biofilm's role in chronic cholesteatomatous otitis media: a pilot study. Otolaryngol Head Neck Surg 2016;154:914-6.

11) Colletti V, Fiorino FG, Carner M, Sacchetto L, Giarbini N. New approach for cochlear implantation: cochleostomy through the middle fossa. Otolaryngol Head Neck Surg 2000;123:467-74.

12) Jang JH, Song JJ, Yoo JC, Lee JH, Oh SH, Chang SO. An alternative procedure for cochlear implantation: transcanal approach. Acta
Otolaryngol 2012;132:845-9.

13) Roehm PC, Gantz BJ. Cochlear implant explantation as a sequela of severe chronic otitis media: case report and review of the literature. Otol Neurotol 2006;27:332-6.

14) Szymański M, Ataide A, Linder T. The use of subtotal petrosectomy in cochlear implant candidates with chronic otitis media. Eur Arch Otorhinolaryngol 2016;273:363-70.

15) Yung M. The use of temporoparietal fascial flap to eliminate wound breakdown in subtotal petrosectomy for chronic discharging ears. Otol Neurotol 2016;37:248-51. 